\title{
Detection of K-complexes and Sleep Spindles (DETOKS) using Sparse Optimization
}

\author{
Ankit Parekh $^{\mathrm{a}, *}$, Ivan W. Selesnick ${ }^{\mathrm{b}}$, David M. Rapoport ${ }^{\mathrm{c}}$, Indu Ayappa ${ }^{\mathrm{c}}$ \\ ${ }^{a}$ Dept. of Mathematics, School of Engineering, New York University \\ ${ }^{b}$ Dept. of Electrical and Computer Engineering, School of Engineering, New York University \\ ${ }^{c}$ Dept. of Medicine, Division of Pulmonary, Critical Care and Sleep Medicine, School of Medicine, New York University
}

\begin{abstract}
Background - This paper addresses the problem of detecting sleep spindles and K-complexes in human sleep EEG. Sleep spindles and K-complexes aid in classifying stage 2 NREM human sleep.

New Method - We propose a non-linear model for the EEG, consisting of a transient, low-frequency, and an oscillatory component. The transient component captures the non-oscillatory transients in the EEG. The oscillatory component admits a sparse time-frequency representation. Using a convex objective function, this paper presents a fast non-linear optimization algorithm to estimate the components in the proposed signal model. The low-frequency and oscillatory components are used to detect K-complexes and sleep spindles respectively.

Results and comparison with other methods - The performance of the proposed method is evaluated using an online EEG database. The F1 scores for the spindle detection averaged $0.70 \pm 0.03$ and the F1 scores for the K-complex detection averaged $0.57 \pm 0.02$. The Matthews Correlation Coefficient and Cohen's Kappa values were in a range similar to the F1 scores for both the sleep spindle and K-complex detection. The F1 scores for the proposed method are higher than existing detection algorithms.
\end{abstract}

Conclusions - Comparable run-times and better detection results than traditional detection algorithms suggests that the proposed method is promising for the practical detection of sleep spindles and K-complexes.

Keywords: Sparse signal, convex optimization, sleep spindle detection, K-complex detection.

\section{Introduction}

Sleep spindles comprise of a group of rhythmic waves that progressively increase and decrease in amplitude [1]. They are of at least 0.5 seconds in duration and have frequencies in the range of $12 \mathrm{~Hz}$ to $14 \mathrm{~Hz}$ [2]. Recent studies have suggested an extended frequency range from $11 \mathrm{~Hz}$ to $16 \mathrm{~Hz}[3,4]$. Sleep spindles are believed to play an important role in synaptic plasticity and memory consolidation during sleep [5]. Alteration in the density of sleep spindles is observed in patients with disorders such as schizophrenia [6], [7], autism [8] and other neurodegenerative and sleep disorders [9]. This leads to mounting belief that sleep spindles may be valuable as diagnostic biomarkers [4].

The K-complex is a transient waveform with a biphasic morphology, characterized by a negative sharp wave followed by a positive one [10]. The K-complex is a relatively large waveform having a duration between 0.5 and 1.5 seconds with an amplitude larger than $75 \mu \mathrm{V}$.

The detection of sleep spindles and K-complexes aid in the scoring of stage N2 of NREM sleep. Traditionally, these morphologically distinct waveforms have been identified manually by trained experts in sleep clinics. This is subjective, time consuming, and prone to errors. The low

${ }^{*}$ Corresponding author. Email address: ankit.parekh@nyu.edu and widely varying inter-rater agreement for sleep spindle and K-complex detection adds to the complexity of the overall scoring process and diagnostic utility. The Cohen's $\kappa$ coefficient for inter-rater manual scoring ranges from 0.46 to 0.89 [11]. Some studies have reported an even lower $\kappa$ coefficient $[12,13]$. Solving the above issues require a reliable automated detector for sleep spindles and $\mathrm{K}$-complexes.

\subsection{Detection Algorithms}

Numerous automated detectors have been developed over the past few years for detecting sleep spindles and $\mathrm{K}$-complexes. At the core of most of the spindle detection algorithms is the use of a constant or adaptive threshold after bandpass filtering the input EEG [7, 14, 15, 16]. Few detectors have been designed to simultaneously detect spindles and K-complexes, whereas most are designed to detect one or the other [17]. The bandpass filter is also excited by transients present in the input EEG. Much of the effort of these algorithms is to either pre-process [18], or post-process [19] the bandpass filtered data, so as to distinguish spindles from transients.

To reduce this overhead, algorithms have been designed using neural networks [20], sleep spindle morphology [21], support vector machines [22], time-frequency methods via the short-time Fourier transform (STFT) $[13,19]$ and adap- 
tive time-frequency methods $[23,24]$. The drawback of using machine learning methods is that they may suffer from over-learning. This increases the number of falsely detected spindles, as compared to scoring by experts (based on the specificity values) [20]. A non-linear pre-processor for the EEG using convex optimization was shown to successfully separate the transients [25]. However, that approach is computationally inefficient as it utilizes two STFT's with high overlap between consecutive windows.

The Teager-Kaiser energy operator (TKEO) has been used for the automatic detection of K-complexes, in conjuction with the time-frequency and neural network methods highlighted above [26, 10, 27]. The TKEO is helpful in extracting the sharp rising and falling edges of the $\mathrm{K}$ complex. However, the presence of transients adversely affects the performance of these algorithms as the TKEO is not able to successfully extract the K-complex activity in the input EEG signal. The spindle and K-complex activity can be made more prominent by suppressing transients in the EEG. However, this suppression is difficult using linear filters, thereby motivating non-linear methods [28].

\subsection{Contribution}

In this paper we propose a non-linear method for the detection of sleep spindles and K-complexes (DETOKS). We model the input EEG as a sum of three components:

1) Transient. The transient component is modeled as a sparse signal possessing a sparse first-order derivative. Essentially, the transient component is comprised of spikes on a baseline of zero.

2) Low-frequency. The low-frequency component of the EEG signal.

3) Oscillations. The rhythmic oscillations in the EEG signal that admit a sparse time-frequency representation.

To estimate the three components from the input EEG signal, we propose an optimization problem utilizing a convex objective function and also derive a fast algorithm for its solution. Post estimation of the components, we use the low-frequency and the oscillatory components for the detection of K-complexes and sleep spindles respectively. Since the proposed method separates the transients from the low-frequency and oscillatory components used for detection, the bandpass filter reveals the spindle activity much more prominently with respect to the baseline. Thus, the proposed approach aims to make traditional spindle detection methods robust by prefacing them with non-linear transient removal.

We list the preliminaries in Section 2 and formulate the DETOKS problem in Section 3. We illustrate the suppression of the transients in the input EEG with various examples in Section 4. We evaluate the performance of DETOKS by applying it on an online EEG database in Section 5. Specifically, we compare spindle and K-complex detection results using the proposed DETOKS method and existing automated detectors [14, 15, 7, 29, 16, 13, 12].

\section{Preliminaries}

\subsection{Notation}

We denote vectors and matrices by lower and upper case letters respectively. The $N$-point signal y is represented by the vector

$$
\mathrm{y}=[y(0), \ldots, y(N-1)]^{T}, \quad \mathrm{y} \in \mathbb{R}^{N},
$$

where $[\cdot]^{T}$ represents the transpose. The $\ell_{1}$ and $\ell_{2}$ norm of the vector $\mathrm{y}$ are defined as

$$
\|\mathrm{y}\|_{1}:=\sum_{n}|y(n)|, \quad\|\mathrm{y}\|_{2}:=\left(\sum_{n}|y(n)|^{2}\right)^{1 / 2} .
$$

The matrix $\mathrm{D}$ is defined as

$$
\mathrm{D}:=\left[\begin{array}{ccccc}
-1 & 1 & & & \\
& -1 & 1 & & \\
& & \ddots & \ddots & \\
& & & -1 & 1
\end{array}\right] .
$$

Using the matrix $\mathrm{D}$, the first order difference of an $N$ point discrete signal $\mathrm{x}$ is given by Dx. Here $\mathrm{D}$ is of size $(N-1) \times N$. The soft-threshold function [30] for $\lambda>$ $0, \lambda \in \mathbb{R}$ is defined as

$$
\operatorname{soft}(x, \lambda):=\left\{\begin{array}{ll}
x-\lambda \frac{x}{|x|}, & |x|>\lambda \\
0, & |x| \leq \lambda,
\end{array} \quad x \in \mathbb{C} .\right.
$$

The soft-threshold function as defined in (4) is valid for complex valued $\mathrm{x}$. The notation $\operatorname{soft}(\mathrm{x}, \lambda)$ implies that the soft-threshold function is applied element-wise to $\mathrm{x}$ with a threshold of $\lambda$. The Teager-Kaiser Energy Operator (TKEO), $T(\cdot)$, for a discrete-time signal y is defined as

$$
[T(\mathrm{y})]_{n}:=y^{2}(n)-y(n-1) \cdot y(n+1) .
$$

\subsection{Sparse Optimization}

To estimate a signal $\mathrm{x}$, possessing a sparse or approximately sparse derivative, from a noisy mixture

$$
\mathrm{y}=\mathrm{x}+\mathrm{w},
$$

it is common to minimize the $\ell_{1}$ norm of the first-order difference Dx subject to a data fidelity constraint [31]. Thus, a suitable optimization problem for estimating $\mathrm{x}$ is

$$
\hat{\mathrm{x}}=\arg \min _{\mathrm{x}}\left\{\frac{1}{2}\|\mathrm{y}-\mathrm{x}\|_{2}^{2}+\lambda\|\mathrm{Dx}\|_{1}\right\} .
$$

The value $\lambda>0$ controls the sparsity of the derivative of $\mathrm{x}$, where the $\ell_{1}$ norm is used as a convex proxy for sparsity. The problem in (7) is known as total variation denoising (TVD) whose solution in linear time is given in [32]. If $\mathrm{x}$ is also sparse, i.e., it comprises spikes on a baseline of zero, 
then an appropriate optimization problem is given by

$$
\hat{\mathrm{x}}=\arg \min _{\mathrm{x}}\left\{\frac{1}{2}\|\mathrm{y}-\mathrm{x}\|_{2}^{2}+\lambda_{1}\|\mathrm{x}\|_{1}+\lambda_{2}\|\mathrm{Dx}\|_{1}\right\} .
$$

This problem is known as the fused lasso [33], [34] and its solution is given by

$$
\mathrm{x}=\operatorname{soft}\left(\operatorname{tvd}\left(\mathrm{y}, \lambda_{2}\right), \lambda_{1}\right)
$$

where $\operatorname{tvd}(\cdot, \cdot)$ represents the solution to the TV denoising problem (7).

\subsection{Short-time Fourier Transform}

Signals that admit a sparse representation can be described by a small number of coefficients using an appropriate transform. Such representations, when they exist, can account for most of the energy contained in a signal [35]. Recently over-complete transforms have been exploited for the sparse representation of signals [36]. A signal consisting of oscillatory pulses can be represented sparsely using the STFT. The STFT requires specification of several parameters such as window length, overlapping factor, and the discrete Fourier transform (DFT) length.

In this work, we use a window length of 1.28 seconds for the STFT and a DFT length equal to the window length. This choice of window length ensures that the DFT length is a power of 2 when the sampling frequency is $50 \mathrm{~Hz}$, $100 \mathrm{~Hz}$, or $200 \mathrm{~Hz}$. In case the EEG is sampled at 128 $\mathrm{Hz}$, we use a one-second window. We use $75 \%$ overlapping between windows, i.e., a hop size of one quarter of the window length. Hence, the STFT is 4-times over-sampled.

Precisely, using a time-frequency array for STFT coefficients of size $M \times K$, for a signal y of length $N$, we define $\Phi: \mathbb{C}^{M \times K} \mapsto \mathbb{C}^{N}$ as

$$
\Phi \mathrm{c}:=\mathrm{STFT}^{-1}(\mathrm{c})
$$

whereas $\Phi^{\mathrm{H}}: \mathbb{C}^{N} \mapsto \mathbb{C}^{M \times K}$ is defined as

$$
\Phi^{\mathrm{H}} \mathrm{y}:=\operatorname{STFT}(\mathrm{y}) .
$$

Using a sine window, we implement the STFT to have the perfect reconstruction property, specifically

$$
\Phi \Phi^{\mathrm{H}}=\mathrm{I}
$$

where $\Phi^{\mathrm{H}}$ represents the Hermitian transpose of $\Phi$ [37].

\section{Simultaneous Detection using DETOKS}

\subsection{Problem Formulation}

We model the EEG signal as

$$
\mathrm{y}=\mathrm{f}+\mathrm{x}+\mathrm{s}+\mathrm{w}, \quad \mathrm{f}, \mathrm{x}, \mathrm{s}, \mathrm{w} \in \mathbb{R}^{N},
$$

where $\mathrm{f}$ represents a low-frequency signal, $\mathrm{x}$ is a sparse signal with sparse first-order derivative, s consists of rhytmic oscillations and is sparse with respect to $\Phi$, and w represents the residual. This kind of a signal model is similar to the one used for transient removal and suppression in $[38,25]$. In contrast to [25], we model the transient component using a sparse first-order derivative rather than an STFT.

We seek estimates for the components $\mathrm{x}, \mathrm{f}$ and $\mathrm{s}$ from the given signal y in (13). The component $\mathrm{s}$ can be modeled as

$$
\mathrm{s}=\Phi \mathrm{c},
$$

where $\mathrm{c} \in \mathbb{C}^{M \times K}$ is the STFT coefficient array. Using a lowpass filter $\mathrm{L}$ we define the highpass filter $\mathrm{H}$ as

$$
\mathrm{H}:=\mathrm{I}-\mathrm{L},
$$

assuming that the frequency response of the lowpass filter is zero-phase or at least approximately zero-phase [38]. For a highpass filter having a $2 d$-order zero at $z=1$, the matrix $\mathrm{H}$ is of size $(N-2 d) \times N$ when applied to a signal of length $N$. Applying the highpass filter to the signal model in (13), we have

$$
\mathrm{H}(\mathrm{y}-\mathrm{x}-\Phi \mathrm{c}) \approx \mathrm{w} .
$$

In order to estimate the components $\mathrm{x}$ and $\mathrm{c}$ from a given $\mathrm{y}$ and minimize the energy of the residual $\mathrm{w}$, we propose the following unconstrained optimization problem,

$$
\begin{gathered}
\arg \min _{\mathrm{x}, \mathrm{c}}\left\{\frac{1}{2}\|\mathrm{H}(\mathrm{y}-\mathrm{x}-\Phi \mathrm{c})\|_{2}^{2}+\lambda_{0}\|\mathrm{x}\|_{1}\right. \\
\left.+\lambda_{1}\|\mathrm{Dx}\|_{1}+\lambda_{2}\|\mathrm{c}\|_{1}\right\} .
\end{gathered}
$$

The objective function in (17) promotes the sparsity of the signal $x$, its first-order derivative Dx, and the STFT coefficient array c, using the $\ell_{1}$ norm. The scalars $\lambda_{0}, \lambda_{1}$ and $\lambda_{2}$ are regularization parameters. We set the highpass filter $\mathrm{H}$ to be a zero-phase recursive discrete-time filter, that we write as

$$
\mathrm{H}=\mathrm{A}^{-1} \mathrm{~B}
$$

where $\mathrm{A}$ and $\mathrm{B}$ are banded ${ }^{1}$ Toeplitz matrices, as described in Sec. VI of [38]. Note that A is of size $(N-2 d) \times(N-$ $2 d)$ and $\mathrm{B}$ is of size $(N-2 d) \times N$, when the $2 d$-order highpass filter is applied to a signal of length $N$. The banded structure of $\mathrm{A}$ and $\mathrm{B}$ aids in the computational efficiency of the algorithm that will be developed in the next sub-section.

\subsection{Algorithm}

Various problems arising in signal processing and biomedical engineering are formulated as convex optimization

\footnotetext{
${ }^{1} \mathrm{~A}$ banded matrix is a type of sparse matrix, whose non-zero entries are restricted on the main diagonal and one or more diagonals on either side of the main diagonal.
} 
problems. The objective function in (17) is also convex, and can be minimized via convex optimization algorithms. There exists a wealth of algorithms for solving problems of type (17) with two or more sparsity inducing penalties $[39,40]$. In particular, we apply Douglas-Rachford splitting [41] to solve (17). The Douglas-Rachford splitting approach results in the well-known alternating direction method of multipliers (ADMM) [42, 43]. Applying variable splitting, we rewrite (17) as

$$
\begin{aligned}
& \arg \min _{\mathrm{u}_{1}, \mathrm{u}_{2}, \mathrm{x}, \mathrm{c}}\{ \frac{1}{2}\left\|\mathrm{H}\left(\mathrm{y}-\mathrm{u}_{1}-\Phi \mathrm{u}_{2}\right)\right\|_{2}^{2}+\lambda_{0}\|\mathrm{x}\|_{1} \\
&\left.+\lambda_{1}\|\mathrm{Dx}\|_{1}+\lambda_{2}\|\mathrm{c}\|_{1}\right\} \\
& \text { s.t. } \quad \mathrm{u}_{1}=\mathrm{x}, \quad \mathrm{u}_{2}=\mathrm{c} .
\end{aligned}
$$

Note that (19) is equivalent to (17). Using the scaled augmented Lagrangian [44], we can minimize (19) by the following iterative procedure.

\section{Repeat:}

$$
\begin{aligned}
\mathrm{u}_{1}, \mathrm{u}_{2} \leftarrow & \arg \min _{\mathrm{u}_{1}, \mathrm{u}_{2}}\left\{\frac{1}{2}\left\|\mathrm{H}\left(\mathrm{y}-\mathrm{u}_{1}-\Phi \mathrm{u}_{2}\right)\right\|_{2}^{2}\right. \\
& \left.+\frac{\mu}{2}\left\|\mathrm{u}_{1}-\mathrm{x}-\mathrm{d}_{1}\right\|_{2}+\frac{\mu}{2}\left\|\mathrm{u}_{2}-\mathrm{c}-\mathrm{d}_{2}\right\|_{2}^{2}\right\}
\end{aligned}
$$

$$
\begin{aligned}
\mathrm{x}, \mathrm{c} \leftarrow & \arg \min _{\mathrm{x}, \mathrm{c}}\left\{\lambda_{0}\|\mathrm{x}\|_{1}+\lambda_{1}\|\mathrm{Dx}\|_{1}+\lambda_{2}\|\mathrm{c}\|_{1}\right. \\
& \left.+\frac{\mu}{2}\left\|\mathrm{u}_{1}-\mathrm{x}-\mathrm{d}_{1}\right\|_{2}+\frac{\mu}{2}\left\|\mathrm{u}_{2}-\mathrm{c}-\mathrm{d}_{2}\right\|_{2}^{2}\right\}
\end{aligned}
$$

$$
\begin{aligned}
& \mathrm{d}_{1} \leftarrow \mathrm{d}_{1}-\left(\mathrm{u}_{1}-\mathrm{x}\right) \\
& \mathrm{d}_{2} \leftarrow \mathrm{d}_{2}-\left(\mathrm{u}_{2}-\mathrm{c}\right)
\end{aligned}
$$

where $\mu>0$. The minimization in (20b) is separable, thus $\mathrm{x}$ and $\mathrm{c}$ can be minimized independently. Thus, we can write (20b) as

$$
\begin{aligned}
& \mathrm{x} \leftarrow \arg \min _{\mathrm{x}}\left\{\lambda_{0}\|\mathrm{x}\|_{1}+\lambda_{1}\|\mathrm{Dx}\|_{1}+\frac{\mu}{2}\left\|\mathrm{u}_{1}-\mathrm{x}-\mathrm{d}_{1}\right\|_{2}\right\} \\
& \mathrm{c} \leftarrow \arg \min _{\mathrm{c}}\left\{\lambda_{2}\|\mathrm{c}\|_{1}+\frac{\mu}{2}\left\|\mathrm{u}_{2}-\mathrm{c}-\mathrm{d}_{2}\right\|_{2}^{2}\right\}
\end{aligned}
$$

The solutions to (21a) and (21b) are readily implemented using the solution to the fused lasso problem as described in Sec. 2.2 and the soft threshold function (4). Specifically, the solutions to (21a) and (21b) are given by

$$
\begin{aligned}
& \mathrm{x} \leftarrow \operatorname{soft}\left(\operatorname{tvd}\left(\mathrm{u}_{1}-\mathrm{d}_{1}, \lambda_{1} / \mu\right), \lambda_{0} / \mu\right), \\
& \mathrm{c} \leftarrow \operatorname{soft}\left(\mathrm{u}_{2}-\mathrm{d}_{2}, \lambda_{2} / \mu\right) .
\end{aligned}
$$

To derive the solution to (20a), we make the following substitutions,

$$
\begin{aligned}
& \mathrm{u}=\left[\mathrm{u}_{1}, \mathrm{u}_{2}\right]^{T}, \quad \mathrm{~d}=\left[\mathrm{d}_{1}, \mathrm{~d}_{2}\right]^{T}, \\
& \overline{\mathrm{x}}=[\mathrm{x}, \mathrm{c}]^{T}, \quad \mathrm{M}=\left[\begin{array}{ll}
\mathrm{I}, & \Phi
\end{array}\right],
\end{aligned}
$$

where $u, d, \bar{x} \in \mathbb{R}^{2 N}$. Due to the substitutions, we rewrite (20a) as

$$
\mathrm{u} \leftarrow \arg \min _{\mathrm{u}}\left\{\frac{1}{2}\|\mathrm{Hy}-\mathrm{HMu}\|_{2}^{2}+\frac{\mu}{2}\|\mathrm{u}-\overline{\mathrm{x}}-\mathrm{d}\|_{2}^{2}\right\} .
$$

The solution to (25) can be written explicitly as

$$
\mathrm{u} \leftarrow\left[\mathrm{M}^{T} \mathrm{H}^{T} \mathrm{HM}+\mu \mathrm{I}_{2 N}\right]^{-1}\left[\left(\mathrm{M}^{T} \mathrm{H}^{T} \mathrm{Hy}+\mu(\overline{\mathrm{x}}+\mathrm{d})\right],\right.
$$

where $\mathrm{I}_{2 N}$ is the $(2 N \times 2 N)$ identity matrix. Due to the form (18) of the highpass filter $\mathrm{H}$ and the perfect reconstruction property (12) of $\Phi$, we have

$$
\begin{aligned}
\mathrm{H}^{T} \mathrm{H} & =\mathrm{B}^{T}\left(\mathrm{AA}^{T}\right)^{-1} \mathrm{~B}, \\
\mathrm{MM}^{T} & =2 \mathrm{I} .
\end{aligned}
$$

Using the matrix inverse lemma [45], (27) and (28), we write

$$
\begin{aligned}
& \left(\mathrm{M}^{T} \mathrm{H}^{T} \mathrm{HM}+\mu \mathrm{I}_{2 N}\right)^{-1} \\
& =\left(\mathrm{M}^{T} \mathrm{~B}^{T}\left(\mathrm{AA}^{T}\right)^{-1} \mathrm{BM}+\mu \mathrm{I}_{2 N}\right)^{-1} \\
& =\frac{1}{\mu}\left(\mathrm{I}_{2 N}-\mathrm{M}^{T} \mathrm{~B}^{T}\left[\mu \mathrm{AA}^{T}+2 \mathrm{BB}^{T}\right]^{-1} \mathrm{BM}\right) .
\end{aligned}
$$

The matrix $\mu \mathrm{AA}^{T}+2 \mathrm{BB}^{T}$ is banded, since the matrices $\mathrm{A}$ and $\mathrm{B}$ are banded. This is advantageous as the iteration now consists of solving a banded system of equations, rather than a dense system in (29). Combining (30) and (26), we obtain the following procedure for solving (20a)

$$
\begin{aligned}
& \mathrm{G} \leftarrow\left(\mu \mathrm{AA}^{T}+2 \mathrm{BB}^{T}\right) \\
& \mathrm{g}_{1} \leftarrow \frac{1}{\mu} \mathrm{B}^{T}\left(\mathrm{AA}^{T}\right)^{-1} \mathrm{By}+\left(\mathrm{x}+\mathrm{d}_{1}\right) \\
& \mathrm{g}_{2} \leftarrow \frac{1}{\mu} \Phi \mathrm{B}^{T}\left(\mathrm{AA}^{T}\right)^{-1} \mathrm{By}+\left(\mathrm{c}+\mathrm{d}_{2}\right) \\
& \mathrm{u}_{1} \leftarrow \mathrm{g}_{1}-\mathrm{B}^{T} \mathrm{G}^{-1} \mathrm{~B}\left(\mathrm{~g}_{1}+\Phi \mathrm{g}_{2}\right) \\
& \mathrm{u}_{2} \leftarrow \mathrm{g}_{2}-\Phi^{\mathrm{H}} \mathrm{B}^{T} \mathrm{G}^{-1} \mathrm{~B}\left(\mathrm{~g}_{1}+\Phi \mathrm{g}_{2}\right)
\end{aligned}
$$

Note that the vector $(1 / \mu) \mathrm{B}^{T}\left(\mathrm{AA}^{T}\right)^{-1} \mathrm{By}$ is used in every iteration. Since the signal $\mathrm{y}$ is not updated in each iteration, we need only compute it once before the loop. Combining the routines discussed above, we obtain the DETOKS algorithm. After we obtain the estimates for $\mathrm{x}$ and $\mathrm{c}$, we calculate the signal components $\mathrm{s}$ and $\mathrm{f}$ as 


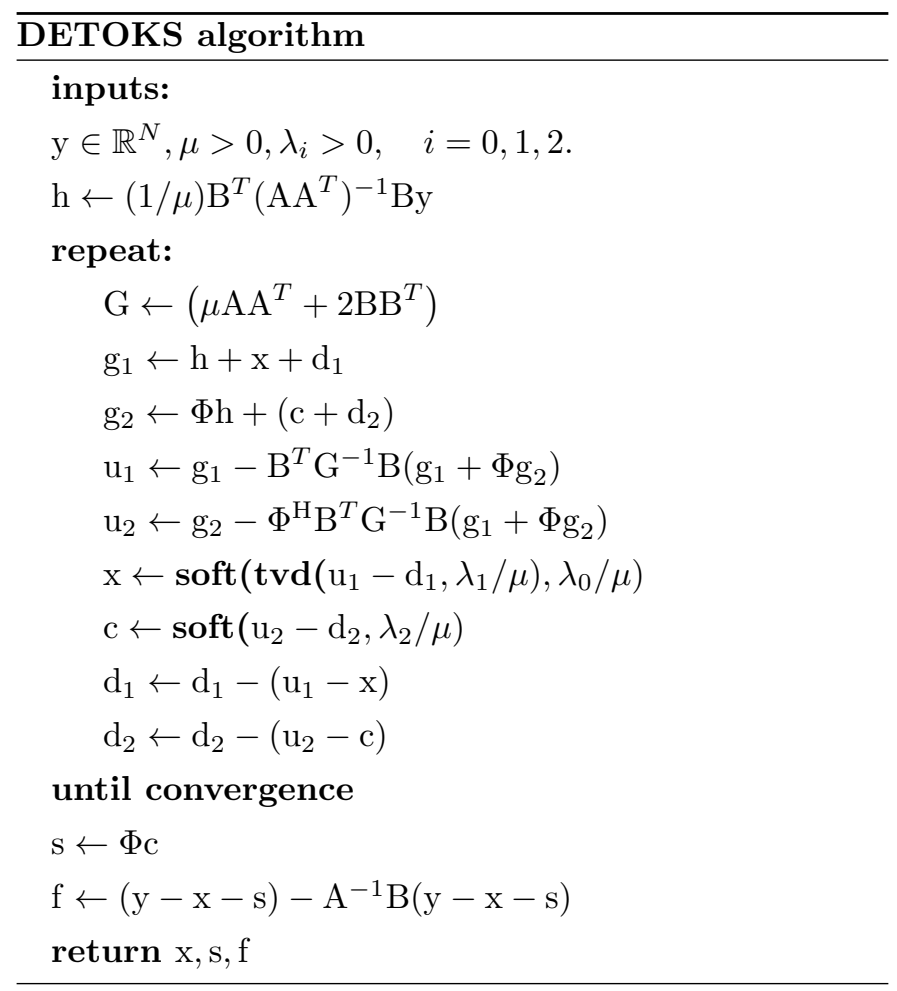

follows

$$
\begin{aligned}
& \mathrm{s} \leftarrow \Phi \mathrm{c} \\
& \mathrm{f} \leftarrow(\mathrm{y}-\mathrm{x}-\mathrm{s})-\mathrm{A}^{-1} \mathrm{~B}(\mathrm{y}-\mathrm{x}-\mathrm{s})
\end{aligned}
$$

where we use the highpass filter defined in (15).

Figure 1 portrays the decomposition of an EEG signal into the signal components $\mathrm{f}, \mathrm{s}$ and $\mathrm{x}$. Notably, the low-frequency component $\mathrm{f}$ contains the K-complex. The oscillatory component $\mathrm{s}$ contains the sleep spindles. The non-oscillatory transients are contained in the sparse component $\mathrm{x}$.

\subsection{Detection of sleep spindles and K-complexes}

The oscillatory component $\mathrm{s}$ is used to detect sleep spindles. We bandpass filter s, to remove any non-spindle oscillations using a 4th order Butterworth filter with a passband of $11.5 \mathrm{~Hz}$ to $15.5 \mathrm{~Hz}$. We denote this bandpass filtered signal as BPF(s). The TKEO $T(\cdot)$, as defined in (5), is then applied to the signal BPF(s). Using a constant value $\left(c_{1}\right)$ for the threshold, a binary signal $b_{\text {spindle }}(t)$, is defined as

$$
\mathrm{b}_{\text {spindle }}(t)= \begin{cases}1, & \mathrm{~T}(\mathrm{BPF}(\mathrm{s}))>c_{1} \\ 0, & \mathrm{~T}(\mathrm{BPF}(\mathrm{s})) \leq c_{1} .\end{cases}
$$

Any detected spindle of duration less than 0.5 seconds is rejected. We allow the maximum duration of a detected spindle to be 3 seconds as in [4].

For detecting K-complexes, we apply the TKEO to the low-frequency component $\mathrm{f}$, and define a binary signal

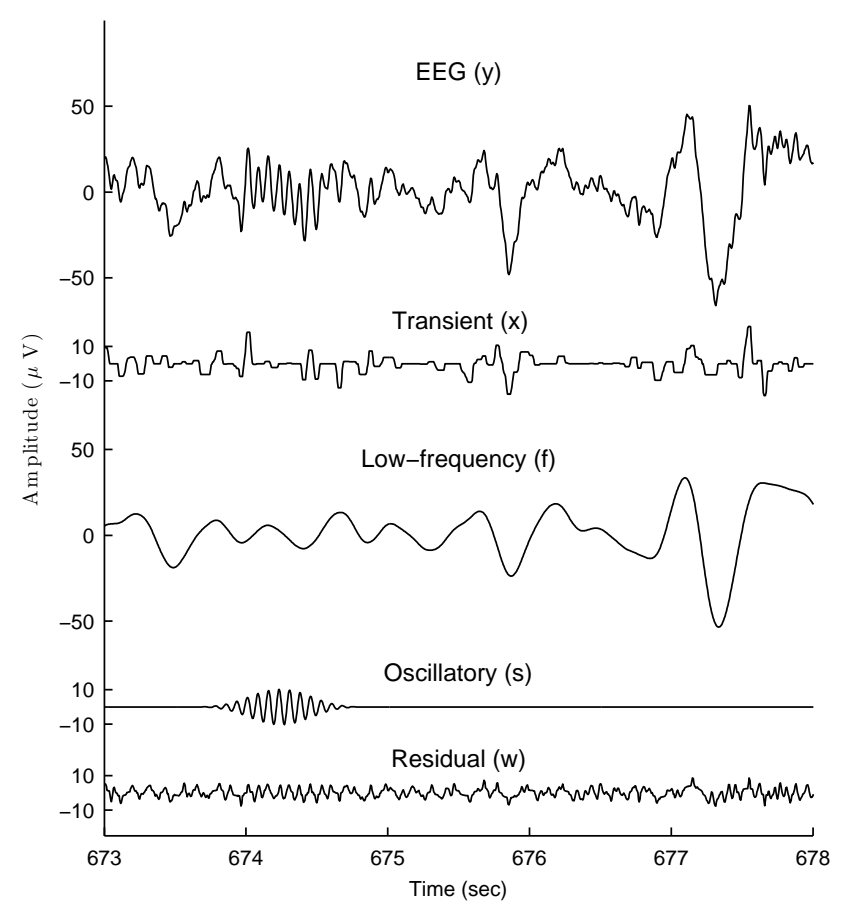

Figure 1: Decomposition of the raw EEG into the signal components $\mathrm{x}, \mathrm{f}$ and $\mathrm{s}$ using the DETOKS algorithm.

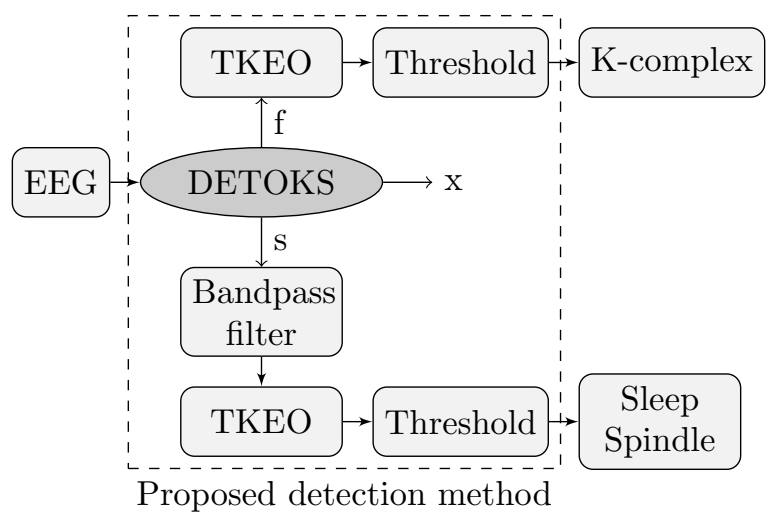

Figure 2: The detection of sleep spindles and K-complexes using the DETOKS algorithm.

$\mathrm{b}_{\mathrm{K}-\text { complex }}(t)$, as in (34), with a constant threshold value $\left(c_{2}\right)$ as

$$
\mathrm{b}_{\text {K-complex }}(t)= \begin{cases}1, & \mathrm{~T}(\mathrm{f})>c_{2} \\ 0, & \mathrm{~T}(\mathrm{f}) \leq c_{2} .\end{cases}
$$

The detected K-complexes of duration less than 0.5 seconds are rejected.

The DETOKS algorithm uses $\mathrm{f}$ and $\mathrm{s}$ to detect sleep spindles and K-complexes simultaneously in sleep EEG. Figure 2 depicts the proposed detection method. Figures 3 and 4 show the detection of a sleep spindle and K-complex respectively using DETOKS. The example EEG shown in Fig. 3 is different from the one in Fig. 4, as the expert annotation for K-complexes was not available for the EEG 


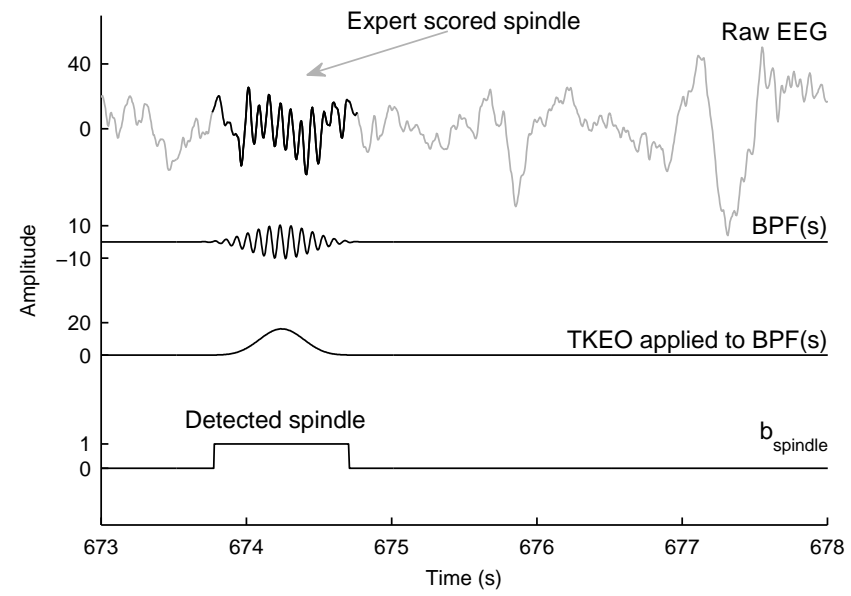

Figure 3: The detection of spindles using a Butterworth bandpass filter (BPF) and the TKEO Operator. The value of the constant threshold $\left(c_{1}\right)$ was fixed at 0.03 .

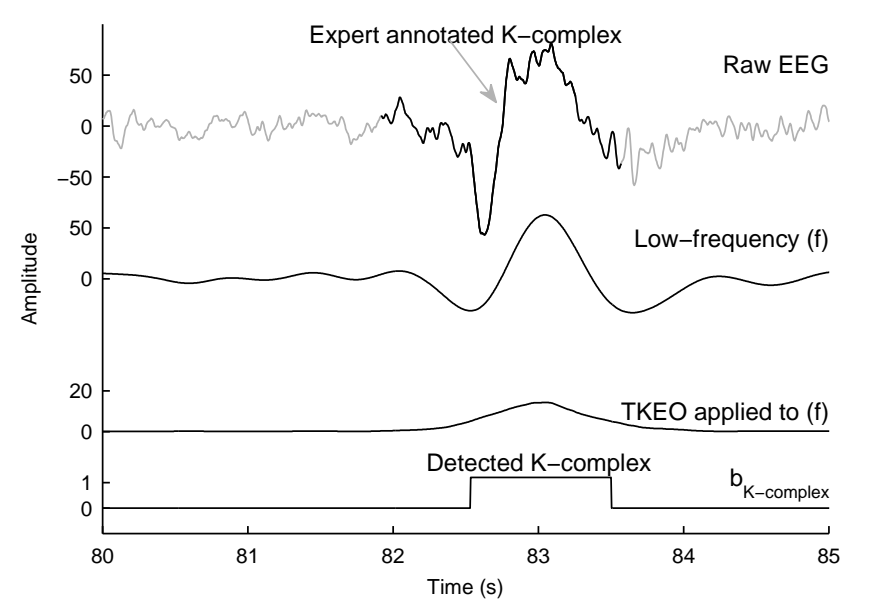

Figure 4: The detection of K-complex using the TKEO. The value of the constant threshold $\left(c_{2}\right)$ was fixed at 1 .

in Fig. 3.

\section{Examples}

We demonstrate the suppression of transients and detection of sleep spindles and K-complexes using DETOKS by applying it to the C3-A1 channel of an EEG. We compare the performance of DETOKS for sleep spindle detection with the methods proposed by Wendt et al. [14] and by Martin et al. [15]. We refer the reader to [4] for a summary of their detection processes.

Recall that the DETOKS algorithm calls for the specification of the parameters $\lambda_{0}, \lambda_{1}, \lambda_{2}$ and $\mu$. The parameters $\lambda_{0}$ and $\lambda_{1}$ influence the sparse nature of the component $\mathrm{x}$ and its derivative Dx respectively. Similarly, $\lambda_{2}$ controls the time-frequency sparsity of the oscillatory component. For the examples in Fig. 1 and the ones that follow, we use $\lambda_{0}=0.6, \lambda_{1}=7$ and $\lambda_{2} \in[7.5,8.5]$. The parameters $\lambda_{0}, \lambda_{1}$, and $\lambda_{2}$ were set empirically to ensure that the

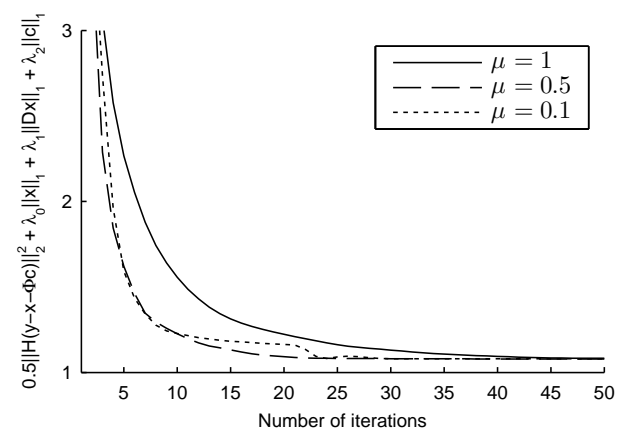

Figure 5: The convergence of the cost function (17) for different values of $\mu$.

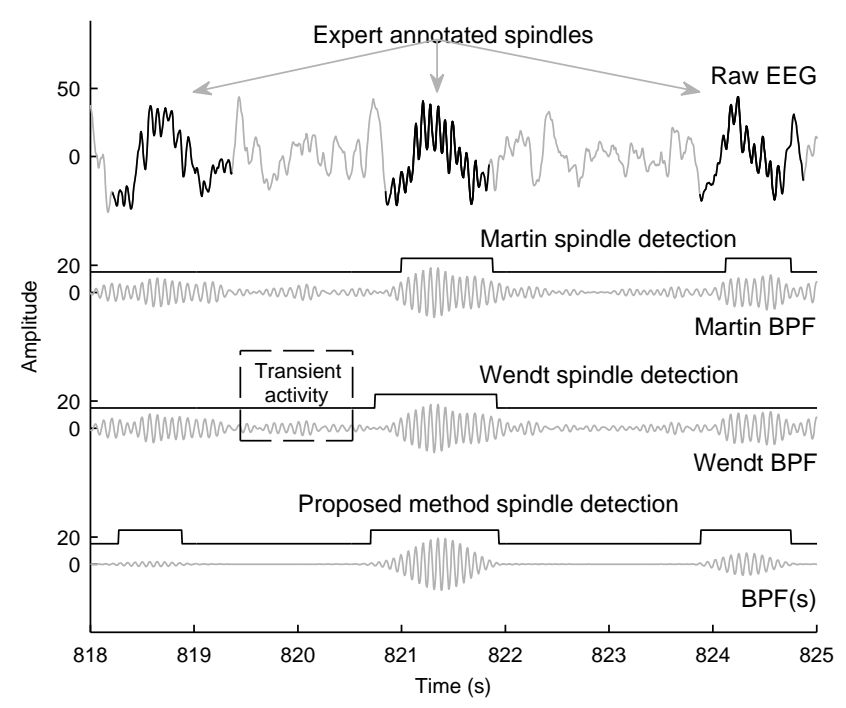

Figure 6: Comparison of the spindle detection using Wendt, Martin and the proposed detection method.

oscillatory component was free of transients and the detected spindle duration nearly matched the duration of a designated spindle. We use threshold values $c_{1}=0.03$ and $c_{2}=1.0$. Although there is no precise definition for the frequency of K-complexes, they are usually within $0.5 \mathrm{~Hz}$ to $4 \mathrm{~Hz}[26,46,47]$. Therefore, we set the lowpass filter cut-off frequency to $4 \mathrm{~Hz}$.

We use the value $\mu=0.5$. This value of $\mu$ gives the lowest value of the objective function in (17) at the 20th iteration (Fig. 5). Note that $\mu$ does not affect the solution to which the DETOKS algorithm converges; it only affects the rate of convergence. Though several parameters must be specified, $\lambda_{2}$ is the only parameter that is varied between $[7.5,8.5]$ by experimental observation in order to emulate the duration of the detected spindle to that of a designated spindle.

\subsection{Sleep Spindle detection}

We compare the spindle detection of the Wendt, Martin, and the proposed DETOKS method in Fig. 6. Experts 


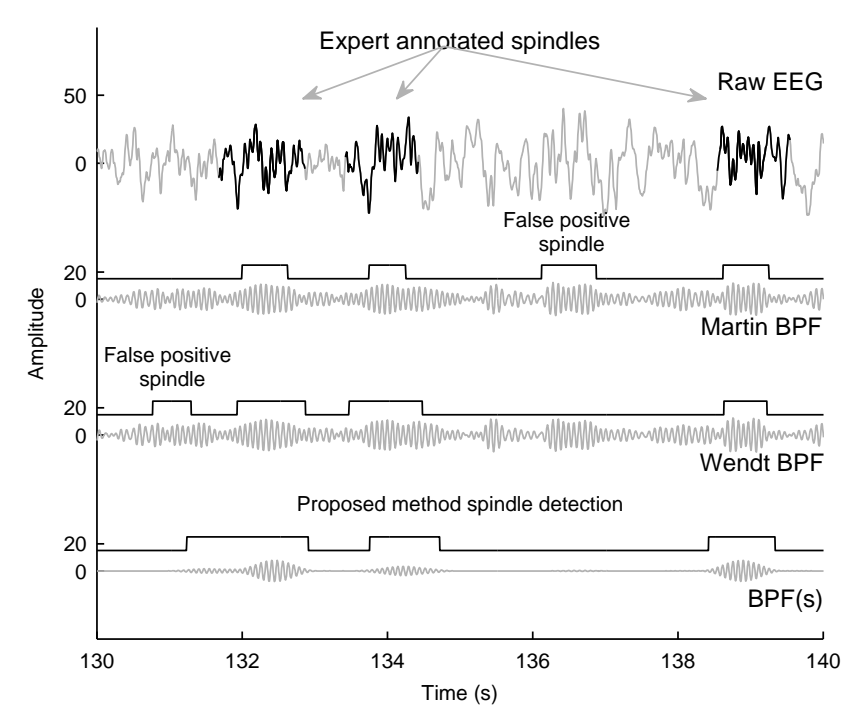

Figure 7: False detection of spindles by the Wendt and Martin algorithm due to the transient activity in the bandpass filtered data.

have annotated three sleep spindles at 818.1, 820.8 and at 823.9 seconds. The Martin algorithm detects only two of the spindles, whereas the Wendt algorithm detects only one spindle. On the other hand, all the three spindles are detected by the proposed detection method. The effect of the suppression of transients can be seen in the bandpass filter activity in Fig. 6. The bandpass filter is excited only during spindle activity in the EEG. In contrast, for the Wendt and Martin algorithms, the low amplitude spindle activity is masked by the transients, which excite the bandpass filter. Thus the suppression of transients increases the number of true positives for spindle detection.

As an another example, consider the issue of falsely detected spindles. Figure 7 illustrates three spindles scored by experts at $131.6,133.4$ and at 138.5 seconds. However, the Martin and Wendt algorithms each detect an additional spindle, which is not annotated by experts. The transient activity excited the bandpass filters again, to the point that the Wendt and Martin algorithms detect false spindles. On the other hand, DETOKS avoids these false detections. Moreover, the duration of the spindles detected by DETOKS better matches those of the experts.

If the parameters $\lambda_{0}, \lambda_{1}$ and $\lambda_{2}$ for the DETOKS algorithm are increased or decreased, then the duration of the spindles detected will also be affected. Thus, one can set the parameters $\lambda_{0}, \lambda_{1}$, and $\lambda_{2}$, so that the duration of a detected spindle emulates the duration of a designated spindle.

\subsection{K-complex detection}

Conventional methods for K-complex detection apply a lowpass filter directly to the EEG then use the TKEO. However, this yields a large number of false positives as seen in Fig. 8. There is only one true positive K-complex at 254.4 seconds and four false positives. Removal of these

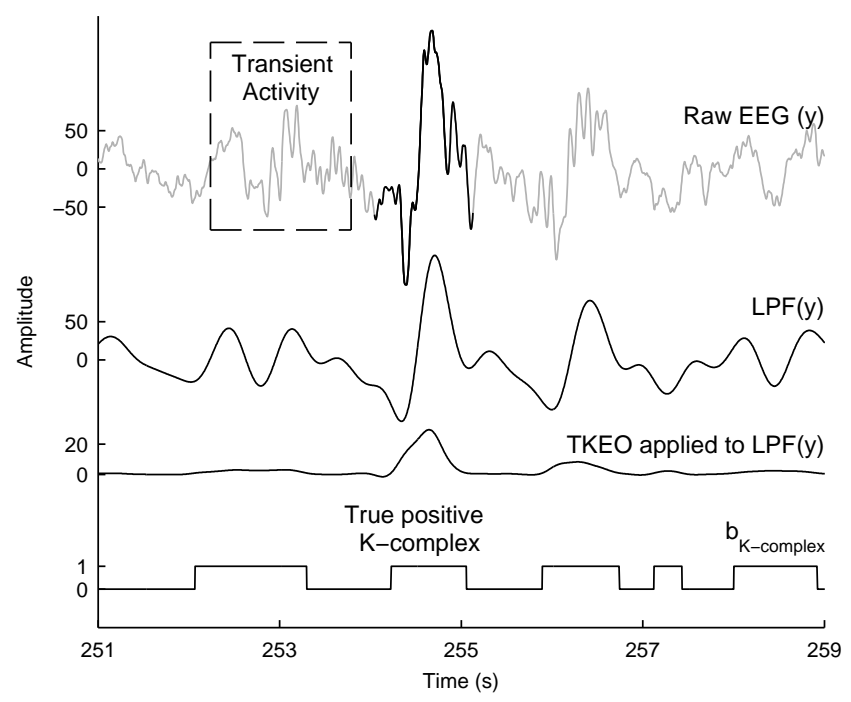

Figure 8: Detecting K-complexes with a 4th order Butterworth lowpass filter $\operatorname{LPF}(\cdot)$ and the TKEO. There are four false positive Kcomplexes and only one true positive.

false positives requires additional processing stages rather than simply using the TKEO on the lowpass filtered EEG [26]. The transients in the EEG excite the lowpass filter, which are then mis-identified as K-complexes after the TKEO stage. Moreover, increasing the threshold for TKEO will not only result in low true positive values, but the duration of the detected K-complexes will also be shorter compared to the expert annotation. In contrast, the component $\mathrm{x}$ obtained using the DETOKS algorithm captures the transient activity, thereby reducing the number of false positives while accurately detecting the designated K-complexes.

Figure 9 compares the K-complexes detected using the proposed method and the method by Devuyst et al. [12]. Specifically, we compare the annotations provided by Devuyst et al. online ${ }^{2}$ for their method, and the K-complex detection by DETOKS. The experts have annotated two K-complexes - at 240 and at 254 seconds. The component $\mathrm{f}$ obtained by the proposed method is also shown. It can be seen that the Devuyst algorithm [12] detects only the K-complex at 255 seconds. Moreover, the duration of the detected K-complex is less than that indicated by the expert. On the other hand, DETOKS detects both K-complexes which better match the expert detection.

\section{Evaluation of DETOKS}

We assess the performance of DETOKS by applying it to an online EEG database. We use two publicly available databases ${ }^{3}$ - one for spindle detection and one for

\footnotetext{
${ }^{2}$ http://www.tcts.fpms.ac.be/ devuyst/Databases/ DatabaseKcomplexes/

${ }^{3}$ http://www.tcts.fpms.ac.be/ devuyst/\#Databases
} 


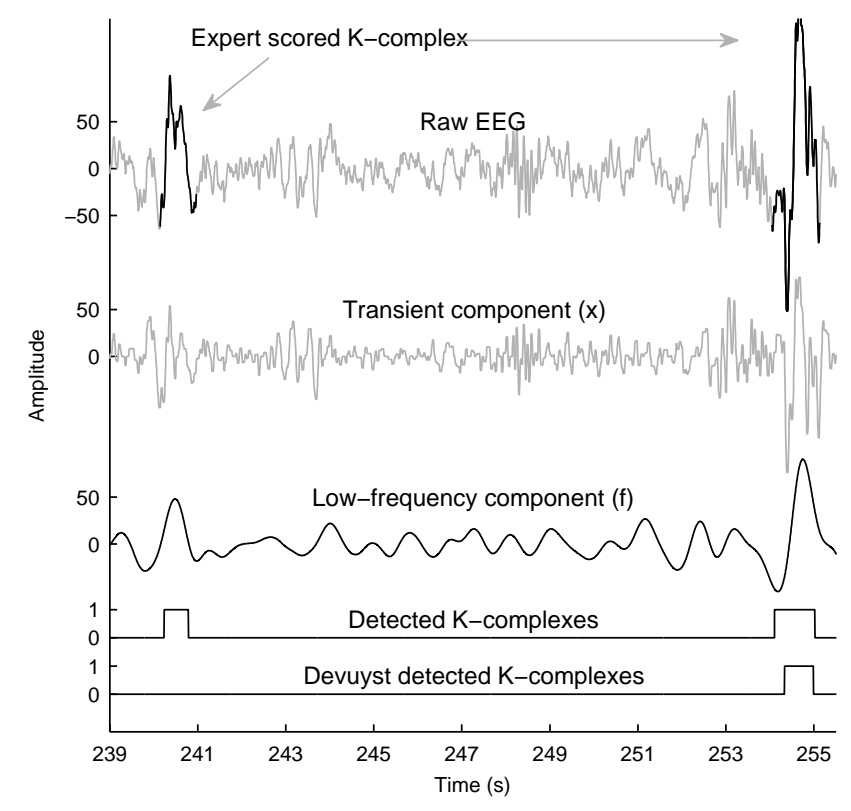

Figure 9: Comparison of the K-complex detection by Devuyst et al. and the proposed simultaneous detection method. The transients in the EEG are captured in the component $\mathrm{x}$.

K-complex detection. The databases ${ }^{4}$ are made available online by Devuyst et al. [13]. For both, spindle and Kcomplex detection, we use the central channel C3-A1 of the EEG. We use these databases for the evaluation study because they are readily available online and provide expert annotations for the scoring of sleep spindles and $\mathrm{K}$ complexes.

\subsection{Database}

As per [13], the EEG database for sleep spindle detection was acquired in a sleep laboratory of a Belgium hospital using a digital 32-channel polygraph (BrainnetTM System of MEDATEC, Brussels, Belgium). The patients possessed different pathologies (dysomnia, restless legs syndrome, insomnia, apnoea/ hypopnoea syndrome) [13]. Three EEG channels (CZ-A1 or C3-A1, FP1-A1, and O1-A1), two EOG channels and one submental EMG channel were recorded. A 30-minute segment of the central EEG channel was extracted from each whole-night recording for sleep spindle scoring. These excerpts were given to two experts who independently scored spindles. Out of the 8 excerpts, we conduct the study using the 5 that were annotated by both experts.

The EEG database for K-complex detection was also acquired in a sleep laboratory of a Belgium hospital using a digital 32-channel polygraph (BrainnetTM System of MEDATEC, Brussels, Belgium) [12]. As in the case of the sleep spindles, a 30-minute segment from each wholenight recording was independently given to two experts

\footnotetext{
${ }^{4}$ University of MONS - TCTS Laboratory (S. Devuyst, T. Dutoit) and Universite Libre de Bruxelles - CHU de Charleroi Sleep Laboratory (M. Kerkhofs)
}

for scoring of K-complexes according to the manual [2] and the recommendations in [12]. For this study, we use the 5 excerpts that were scored by both experts.

\subsection{Existing detection methods}

For sleep-spindle detection, we compare DETOKS to the existing algorithms by Wendt et al. (S1) [14], Martin et al. (S2) [15], Wamsley et al. (S3) [7], Bòdizs et al. (S3) [29], Mölle et al. (S3) [16], and Devuyst et al. (S6) [13]. A summary of these algorithms is provided in $[4,13]$. We reject any spindles detected by S1-S6 of less than 0.5 seconds in duration. For K-complex detection we compare DETOKS to the annotations provided by Devuyst et al. for their detection method [12].

\subsection{Measure of Performance}

We use the detection by experts, for the sleep spindles and the K-complexes, as the gold standard. A sample point of the EEG is recorded as a sleep spindle or a $\mathrm{K}$ complex if it was scored as such by either expert. This is the by-sample analysis of a detector [4] using a 'union' rule of the expert detection [13]. We create a contingency table, for sleep spindles and K-complexes, to calculate the values of true positive (TP), false positive (FP), true negative $(\mathrm{TN})$ and false negative $(\mathrm{FN})$. These values are then used to calculate the recall and precision values for the detector. We use $\mathrm{F}_{1}$ scores to evaluate the detectors. The $\mathrm{F}_{1}$ score ranges from 0 to 1 , with 1 denoting the perfect detector. Using the contingency table, the statistical measures of Cohen's $\kappa$ [48] and Matthews Correlation Coefficient (MCC) [49] can also be calculated.

\subsection{Results}

The C3-A1 channel of the EEG was processed using the proposed DETOKS algorithm and the estimates of the components s and $\mathrm{f}$ were then used for sleep spindle and K-complex detection respectively. We used the same parameters for $\lambda_{0}, \lambda_{1}, \lambda_{2}$ and the threshold values $c_{1}$ and $c_{2}$ as in Sec. 4. We used the STFT parameters given in Sec. 2.3.

Figure 10 displays the $\mathrm{F}_{1}$ scores for sleep spindle detection, recall, and precision values for the algorithms in Sec. 5.2 and DETOKS. Table 1 lists the $\mathrm{F}_{1}$ scores for K-complex detection for the Devuyst algorithm [12] and DETOKS. The recall and precision values are also listed.

Further statistical measures of performance are listed in the Appendix. The proposed DETOKS method takes about 4 minutes to run 20 iterations on approximately 8 hours of EEG at sampling frequency $100 \mathrm{~Hz}$.

\subsection{Discussions}

The proposed DETOKS method achieves high $\mathrm{F}_{1}$ scores for spindle detection due to the suppression of transients. As seen in Appendix A, the values of $\kappa$ and MCC were similar to the $\mathrm{F}_{1}$ scores obtained. The TP values are comparatively higher than those of algorithms S1-S6. The number 


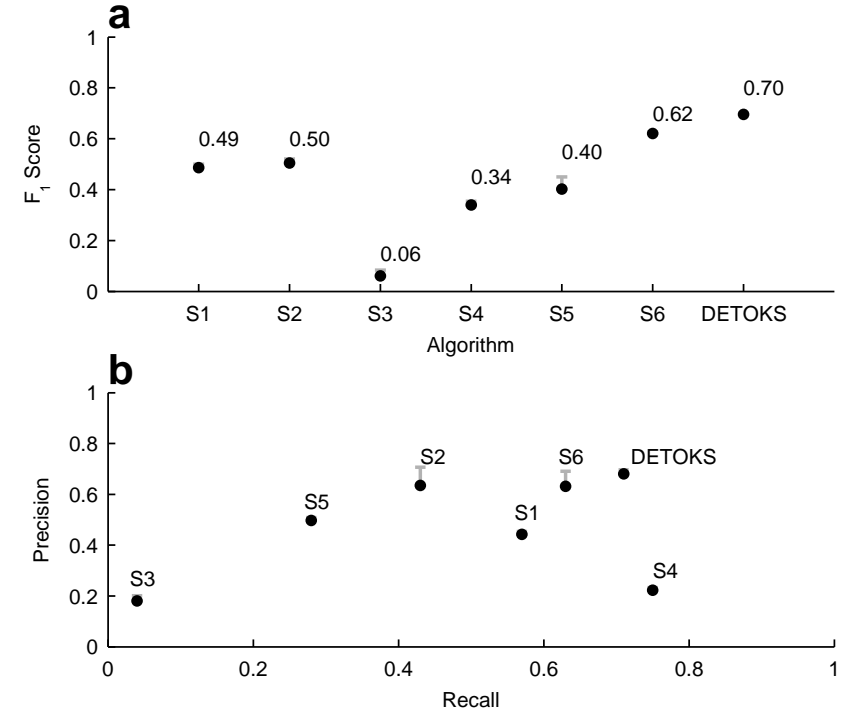

Figure 10: Results of the evaluation study in Sec. 5. (a) Comparison of the $\mathrm{F}_{1}$ scores of the spindle detection algorithms. (b) Recall and Precision values for the algorithms S1-S6 and the proposed DETOKS method. The error bars represent standard error of the mean.

\begin{tabular}{lrr}
\hline & Devuyst et al. & DETOKS \\
\cline { 2 - 3 } $\mathrm{F}_{1}$ Score & 0.51 & 0.57 \\
Recall & 0.40 & 0.61 \\
Precision & 0.74 & 0.56 \\
\hline
\end{tabular}

Table 1: K-complex detection performance evaluation

of false positives are also reduced. The reduction in FP values and an increase in TP values, result in the $\mathrm{F}_{1}$ score of the proposed detection method for sleep spindles averaging $0.7 \pm 0.03$ over the five excerpts. Note that an extensive study on sleep spindle detection by automated detectors [4] showed that the $\mathrm{F}_{1}$ score of 24 individual experts averaged $0.69 \pm 0.06$ on their crowd-sourcing generated spindle gold standard. The proposed DETOKS method achieves the average $\mathrm{F}_{1}$ score of an expert consistently, thus making it a reliable spindle detector.

A balanced detector shows high recall and precision values. Figure 10 shows that DETOKS is the most balanced among the 6 algorithms S1-S6 for spindle detection. The recall and precision values for DETOKS averaged $0.71 \pm 0.01$ and $0.68 \pm 0.03$ respectively. The algorithm by Bòdizs et al. [29] has a higher recall than the DETOKS but low precision, indicating the detection of a large number of false positives in contrast to DETOKS. The proposed DETOKS method is not only reliable but also a balanced spindle detector.

For the detection of K-complexes, the proposed method obtained an average $\mathrm{F}_{1}$ score of $0.57 \pm 0.019$ over the 5 excerpts. The performance of DETOKS for K-complex detection was also balanced compared to the Devuyst algorithm [12]. It can be seen in Table 1, that the proposed

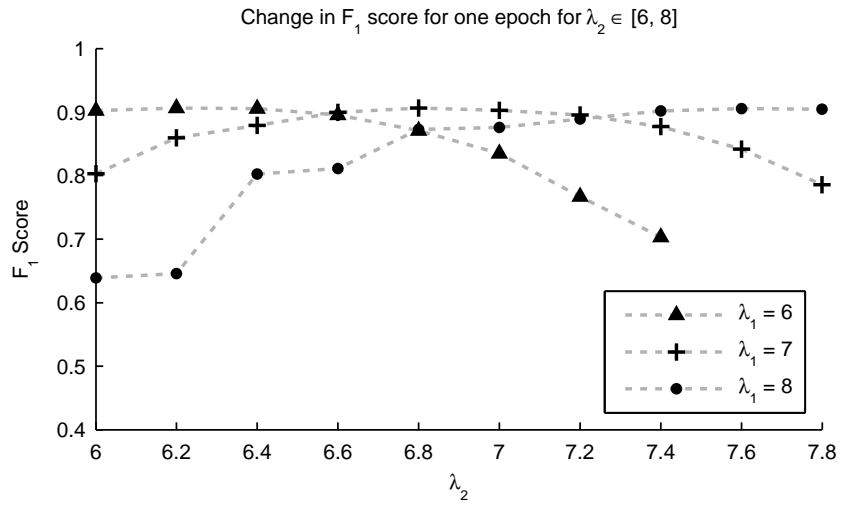

Figure 11: Change in the $F_{1}$ score of an epoch on changing the parameters $\lambda_{1}$ and $\lambda_{2}$ in (17). $\lambda_{0}$ is fixed at 0.6 .

detection method has better recall and precision values compared to the Devuyst algorithm. Note that the recall, precision, and $\mathrm{F}_{1}$ scores for the Devuyst method were calculated based on the TP, FP, TN, and FN values provided in $[12]$.

The algorithm by Devuyst et al. employs K-complex detection using a training dataset [12]. It tunes the detection process, based on visually identified K-complexes. In contrast, the proposed detection method needs no training data-set. Moreover, for different databases, based on the sampling frequency, the only parameters that need to be tuned are the regularization parameters $\lambda_{0}, \lambda_{1}$ and $\lambda_{2}$. The other parameters, for example the STFT window length, are based on the definition of sleep spindles and hence need no tuning. Figure 11 shows that the $\mathrm{F}_{1}$ score for sleep spindle detection for one EEG epoch is affected marginally when the parameters $\lambda_{1}$ and $\lambda_{2}$ are changed in small increments, with $\lambda_{0}$ fixed. Similar change is observed when $\lambda_{0}$ is varied in addition to $\lambda_{1}$ and $\lambda_{2}$.

\section{Conclusion}

This paper proposes an EEG signal model comprising of 1) a transient component, 2) a low-frequency component, and 3) an oscillatory component with sparse timefrequency representation. We propose a convex optimization algorithm for the detection of K-complexes and sleep spindles (DETOKS), which estimates the three components in the signal model. The proposed DETOKS method utilizes the Teager Kaiser Energy Operator (TKEO) as a means to obtain the envelope of the bandpass filtered oscillatory component and the low-frequency component. The envelopes are then used for the detection of sleep spindles and K-complexes.

An assessment of the proposed DETOKS method yields $\mathrm{F}_{1}$ scores of average $0.70 \pm 0.03$ for sleep spindle detection and $0.57 \pm 0.019$ for K-complex detection. The proposed signal model and the DETOKS algorithm give better results for the detection of both sleep spindles and K-complexes, compared to existing algorithms specifically 
aimed at one or the other. The average $\mathrm{F}_{1}$ score of the proposed detector is about the same as the $F_{1}$ score attained by individual experts annotating crowd-sourced spindle data [4]. Comparable run-times and better detection results than traditional spindle and K-complex detection algorithms suggest that the proposed DETOKS method is practical for the detection of sleep spindles and K-complexes.

\section{References}

[1] M. H. Silber, S. Ancoli-Israel, M. H. Bonnet, S. Chokroverty, M. M. Grigg-Damberger, M. Hirshkowitz, S. Kapen, S. a. Keenan, M. H. Kryger, T. Penzel, M. R. Pressman, C. Iber, The visual scoring of sleep in adults., Journal of clinical sleep medicine : JCSM : official publication of the American Academy of Sleep Medicine 3 (2) (2007) 121-31, ISSN 1550-9389.

[2] R. Berry, R. Brooks, C. Gamaldo, S. Harding, R. Lloyd, C. Marcus, B. Vaughn, The AASM Manual for the Scoring of Sleep and Associated Events: Rules, Terminology and Technical Specifications, 2013.

[3] S. Devuyst, T. Dutoit, J. Didier, F. Meers, E. Stanus, P. Stenuit, M. Kerkhofs, Automatic sleep spindle detection in patients with sleep disorders, Proc. IEEE Int. Conf. Eng. in Medicine and Biology (EMBC) (2006) 3883-3886ISSN 1557-170X, doi: 10.1109/IEMBS.2006.259298.

[4] S. C. Warby, S. L. Wendt, P. Welinder, E. G. S. Munk, O. Carrillo, H. B. D. Sorensen, P. Jennum, P. E. Peppard, P. Perona, E. Mignot, Sleep-spindle detection: crowdsourcing and evaluating performance of experts, non-experts and automated methods., Nature methods 11 (4) (2014) 385-92, ISSN 1548-7105, doi:10.1038/nmeth. 2855 .

[5] S. M. Fogel, C. T. Smith, The function of the sleep spindle: a physiological index of intelligence and a mechanism for sleep-dependent memory consolidation., Neuroscience and biobehavioral reviews 35 (5) (2011) 1154-65, ISSN 1873-7528, doi:10.1016/j.neubiorev.2010.12.003.

[6] F. Ferrarelli, R. Huber, M. J. Peterson, M. Massimini, M. Murphy, B. a. Riedner, A. Watson, P. Bria, G. Tononi, Reduced sleep spindle activity in schizophrenia patients., The American journal of psychiatry 164 (3) (2007) 483-92, ISSN 0002-953X, doi:10.1176/appi.ajp.164.3.483.

[7] E. Wamsley, M. Tucker, A. Shinn, Reduced sleep spindles and spindle coherence in schizophrenia: mechanisms of impaired memory consolidation?, Biol. Psychiatry 71 (2) (2012) 154-161, doi:10.1016/j.biopsych.2011.08.008.Reduced.

[8] E. Limoges, L. Mottron, C. Bolduc, C. Berthiaume, R. Godbout, Atypical sleep architecture and the autism phenotype., Brain : a journal of neurology 128 (Pt 5) (2005) 1049-61, ISSN 1460-2156, doi:10.1093/brain/awh425.

[9] D. Petit, J. F. Gagnon, M. L. Fantini, L. Ferini-Strambi, J. Montplaisir, Sleep and quantitative EEG in neurodegenerative disorders, doi:10.1016/j.jpsychores.2004.02.001, 2004.

[10] C. Richard, R. Lengelle, Joint time and time-frequency optimal detection of K-complexes in sleep EEG., Computers and biomedical research, an international journal 31 (1998) 209-229, ISSN 0010-4809, doi:S0010480998914768[pii].

[11] C. Stepnowsky, D. Levendowski, D. Popovic, I. Ayappa, D. M. Rapoport, Scoring accuracy of automated sleep staging from a bipolar electroocular recording compared to manual scoring by multiple raters., Sleep medicine 14 (11) (2013) 1199-207, ISSN 1878-5506, doi:10.1016/j.sleep.2013.04.022.

[12] S. Devuyst, T. Dutoit, P. Stenuit, M. Kerkhofs, Automatic Kcomplexes detection in sleep EEG recordings using likelihood thresholds, in: Proc. IEEE Int. Conf. Eng. in Medicine and Biology (EMBC), ISBN 9781424441235, ISSN 1557-170X, 46584661, doi:10.1109/IEMBS.2010.5626447, 2010.

[13] S. Devuyst, T. Dutoit, P. Stenuit, M. Kerkhofs, Automatic sleep spindles detection - overview and development of a stan- dard proposal assessment method., Proc. IEEE Int. Conf. Eng. in Medicine and Biology (EMBC) (2011) 1713-1716ISSN 1557170X, doi:10.1109/IEMBS.2011.6090491.

[14] S. L. Wendt, J. E. Christensen, J. Kempfner, H. L. Leonthin, P. Jennum, H. B. D. Sorensen, Validation of a novel automatic sleep spindle detector with high performance during sleep in middle aged subjects, Proc. IEEE Int. Conf. Eng. in Medicine and Biology (EMBC) (2012) 4250-4253ISSN 1557-170X, doi: 10.1109/EMBC.2012.6346905.

[15] N. Martin, M. Lafortune, J. Godbout, M. Barakat, R. Robillard, G. Poirier, C. Bastien, J. Carrier, Topography of age-related changes in sleep spindles., Neurobiology of aging 34 (2) (2013) 468-76, ISSN 1558-1497, doi:10.1016/j.neurobiolaging.2012.05. 020 .

[16] S. Gais, M. Mölle, K. Helms, J. Born, Learning-dependent increases in sleep spindle density., The Journal of neuroscience : the official journal of the Society for Neuroscience 22 (15) (2002) 6830-4, ISSN 1529-2401, doi:20026697.

[17] T. A. Camilleri, K. P. Camilleri, S. G. Fabri, Automatic detection of spindles and K-complexes in sleep EEG using switching multiple models, Biomedical Signal Processing and Control 10 (2014) 117-127, ISSN 17468094, doi:10.1016/j.bspc.2014.01. 010 .

[18] A. Jaleel, B. Ahmed, R. Tafreshi, D. B. Boivin, L. Streletz, N. Haddad, Improved spindle detection through intuitive preprocessing of electroencephalogram., Journal of neuroscience methods 233 (2014) 1-12, ISSN 1872-678X, doi:10.1016/j. jneumeth.2014.05.009.

[19] S. Motamedi-Fakhr, M. Moshrefi-Torbati, M. Hill, C. M. Hill, P. R. White, Signal processing techniques applied to human sleep EEG signals: A review, Biomedical Signal Processing and Control 10 (2014) 21-33, ISSN 17468094, doi:10.1016/j.bspc. 2013.12.003.

[20] D. Gorur, U. Halici, H. Aydin, Sleep spindles detection using short time Fourier transform and neural networks, Int. Jt. Conf. Neural Networks (IJCNN) (2002) 1631-1636.

[21] J. Costa, M. Ortigueira, A. Batista, L. Paiva, An automatic sleep spindle detector based on WT, STFT and WMSD, World Academy of Science, Engineering and Technology (2012) 18331836.

[22] N. Acir, C. Güzeli, Automatic recognition of sleep spindles in EEG via radial basis support vector machine based on a modified feature selection algorithm, Neural Computing and Applications 14 (1) (2005) 56-65, ISSN 0941-0643, doi:10.1007/ s00521-004-0442-z.

[23] P. Durka, K. Blinowska, Matching pursuit parametrization of sleep spindles, Engineering in Medicine and Biology 3 (1996) 1011-1012, doi:10.1109/IEMBS.1996.652685.

[24] P. Durka, D. Ircha, K. Blinowska, Stochastic time-frequency dictionaries for matching pursuit, IEEE Transactions on Signal Processing 49 (3) (2001) 507-510, ISSN 1053587X, doi:10.1109/ 78.905866 .

[25] A. Parekh, I. W. Selesnick, D. M. Rapoport, I. Ayappa, Sleep spindle eetection using time-frequency sparsity, in: Proc. IEEE Signal Processing in Medicine and Biology Symposium (SPMB), doi:10.1109/SPMB.2014.7002965, 2014.

[26] A. Erdamar, F. Duman, S. Yetkin, A wavelet and teager energy operator based method for automatic detection of K-Complex in sleep EEG, Expert Systems with Applications 39 (1) (2012) 1284-1290, ISSN 09574174, doi:10.1016/j.eswa.2011.07.138.

[27] C. Strungaru, M. S. Popescu, Neural network for sleep EEG K-complex detection., Biomedizinische Technik. Biomedical engineering 43 Suppl 3 (1998) 113-116, ISSN 0013-5585, doi: 10.1515/bmte.1998.43.s3.113.

[28] I. W. Selesnick, Resonance-based signal decomposition: A new sparsity-enabled signal analysis method, Signal Processing 91 (12) (2011) 2793-2809, ISSN 01651684, doi:DOI:10.1016/j. sigpro.2010.10.018.

[29] R. Bódizs, J. Körmendi, P. Rigó, A. S. Lázár, The individual adjustment method of sleep spindle analysis: methodological improvements and roots in the fingerprint paradigm., Journal 
of neuroscience methods 178 (1) (2009) 205-13, ISSN 0165-0270, doi:10.1016/j.jneumeth.2008.11.006.

[30] D. L. Donoho, De-noising by soft-thresholding, IEEE Transactions on Information Theory 41 (1995) 613-627, ISSN 00189448, doi:10.1109/18.382009.

[31] L. I. Rudin, S. Osher, E. Fatemi, Nonlinear total variation based noise removal algorithms, Physica D: Nonlinear Phenomena 60 (1992) 259-268, ISSN 01672789, doi:10.1016/0167-2789(92) 90242-F.

[32] L. Condat, A Direct Algorithm for 1-D Total Variation Denoising, IEEE Signal Processing Letters 20 (11) (2013) 1054-1057, ISSN 1070-9908, doi:10.1109/LSP.2013.2278339.

[33] A. Rinaldo, Properties and refinements of the fused lasso, The Annals of Statistics 37 (5B) (2009) 2922-2952, ISSN 0090-5364, doi:10.1214/08-AOS665.

[34] R. Tibshirani, M. Saunders, S. Rosset, J. Zhu, K. Knight, Sparsity and smoothness via the fused lasso, Journal of the Royal Statistical Society. Series B: Statistical Methodology 67 (2005) 91-108, ISSN 13697412, doi:10.1111/j.1467-9868.2005.00490.x.

[35] S. Mallat, Chapter 1 - Sparse Representations, in: A wavelet tour of signal processing: The sparse way, Academic Press, ISBN 978-0-12-374370-1, 1-31, doi:http://dx.doi.org/10.1016/ B978-0-12-374370-1.00005-7, 2009.

[36] S. Chen, D. L. Donoho, M. A. Saunders, Atomic decomposition by basis pursuit, SIAM journal on scientific computing 43 (1) (1998) 129-159, doi:10.1137/S1064827596304010.

[37] I. W. Selesnick, Short-time Fourier transform and speech denoising, http://cnx.org/content/m32294/ .

[38] I. W. Selesnick, H. L. Graber, D. S. Pfeil, R. L. Barbour, Simultaneous low-pass filtering and total variation denoising, IEEE Transactions on Signal Processing 62 (5) (2014) 1109-1124, ISSN NA, doi:10.1109/TSP.2014.2298836.

[39] A. Chambolle, T. Pock, A first-order primal-dual algorithm for convex problems with applications to imaging, J. Math. Vis. 40 (1) (2011) 120-145, ISSN 09249907, doi:10.1007/ s10851-010-0251-1.

[40] P. L. Combettes, J.-C. Pesquet, Proximal thresholding algorithm for minimization over orthonormal bases, SIAM Journal on Optimization 18 (4) (2007) 1351-1376, ISSN 1052-6234, doi: $10.1137 / 060669498$.

[41] P. L. Combettes, J.-C. Pesquet, Proximal splitting methods in signal processing, in: H. H. Bauschke, Others (Eds.), FixedPoint Algorithms for Inverse Problems in Science and Engineering, Springer-Verlag, 185-212, 2011.

[42] M. Afonso, J. Bioucas-Dias, M. Figueiredo, Fast image recovery using variable splitting and constrained optimization., IEEE Transactions on Image Processing 19 (2010) 2345-2356, ISSN 1941-0042, doi:10.1109/TIP.2010.2047910.

[43] D. Gabay, Applications of the method of multipliers to varitional inequalities, in: Augmented Lagrangian methods: Applications to the numerical solution of boundary-value problems, Elsevier, 299-331, 1983.

[44] S. Boyd, N. Parikh, E. Chu, J. Eckstein, Distributed Optimization and Statistical Learning via the Alternating Direction Method of Multipliers, Foundations and Trends in Machine Learning 3 (1) (2010) 1-122, ISSN 1935-8237, doi:10.1561/ 2200000016.

[45] D. Tylavsky, G. Sohie, Generalization of the matrix inversion lemma, Proceedings of the IEEE 74 (1986) 1050-1052, ISSN 0018-9219, doi:10.1109/PROC.1986.13587.

[46] G. Bremer, J. R. Smith, I. Karacan, Automatic detection of the $\mathrm{K}$-complex in sleep electroencephalograms., IEEE transactions on bio-medical engineering 17 (1970) 314-323, ISSN 0018-9294, doi:10.1109/TBME.1970.4502759.

[47] M. Woertz, T. Miazhynskaia, P. Anderer, G. Dorffner, Automatic K-complex detection: comparison of two different approaches, Abstracts of the ESRS, JSR 13 (1).

[48] J. Cohen, A coefficient of agreement for nominal scales, Educational and Psychological Measurement 20 (1) (1960) 37-46, doi:10.1177/001316446002000104.

[49] B. W. Matthews, Comparison of the predicted and observed secondary structure of T4 phage lysozyme, Biochimica et Biophysica Acta (BBA) - Protein Structure 405 (2) (1975) 442-451, doi:10.1016/0005-2795(75)90109-9.

\section{Appendix A. K-complex detection}

Performance and comparison of the proposed method for K-complex detection with Devuyst et al.

\begin{tabular}{|c|c|c|c|c|c|c|c|c|c|c|}
\hline & \multicolumn{2}{|c|}{ Excerpt 1} & \multicolumn{2}{|c|}{ Excerpt 2} & \multicolumn{2}{|c|}{ Excerpt 3} & \multicolumn{2}{|c|}{ Excerpt 4} & \multicolumn{2}{|c|}{ Excerpt 5} \\
\hline & Devuyst & DETOKS & Devuyst & DETOKS & Devuyst & DETOKS & Devuyst & DETOKS & Devuyst & DETOKS \\
\hline & 2646 & 4777 & 4800 & 7824 & 1676 & 1973 & 6672 & 10404 & 4840 & 7140 \\
\hline & 349221 & 341758 & 348236 & 343917 & 355906 & 355150 & 335746 & 331796 & 347611 & 345900 \\
\hline & 627 & 8089 & 1337 & 5655 & 889 & 1644 & 2674 & 6623 & 1869 & 3580 \\
\hline & 7506 & 5375 & 5627 & 2603 & 1529 & 1232 & 14908 & 11176 & 5680 & 3380 \\
\hline ecall & 0.261 & 0.471 & 0.460 & 0.750 & 0.523 & 0.616 & 0.309 & 0.482 & 0.460 & 0.679 \\
\hline recision & 0.808 & 0.371 & 0.782 & & 0.653 & 0.545 & 0.714 & 0.611 & 0.721 & 0.666 \\
\hline $5 \mathrm{~s}$ & 0.394 & 0.415 & 0.5 & & 0.581 & 0.578 & & 0.539 & 0.562 & 0.672 \\
\hline pecificity & 0.998 & 0.977 & 0.996 & 0.984 & 0.998 & 0.995 & 0.992 & 0.980 & 0.995 & 0.990 \\
\hline IPV & 0.979 & 0.985 & 0.984 & 0.992 & 0.996 & 0.997 & 0.957 & 0.967 & 0.984 & 0.990 \\
\hline curacy & 0.9 & & & & & 0.992 & & 0.951 & 0.979 & 0.981 \\
\hline ppa & 0.386 & 0.396 & 0.570 & 0.643 & 0.578 & 0.574 & 0.410 & 0.513 & 0.552 & 0.652 \\
\hline & 0.451 & 0.399 & 0.591 & 0.649 & 0.581 & 0.575 & 0.450 & 0.517 & 0.566 & 0.661 \\
\hline
\end{tabular}




\section{Appendix B. Sleep spindle detection}

Performance of the proposed DETOKS method for spindle detection and comparison with other widely used algorithms

\begin{tabular}{|c|c|c|c|c|c|c|c|}
\hline Excerpt 1 & Wendt & Martin & Wamsley & Bodizs & Moelle & Devuyst & DETOKS \\
\hline TP & 8357 & 4121 & 2374 & 9550 & 4991 & 9692 & 9354 \\
\hline TN & 156774 & 164771 & 166242 & 137540 & 164911 & 159737 & 162632 \\
\hline FP & 9751 & 1754 & 283 & 28985 & 1614 & 6788 & 3892 \\
\hline $\mathrm{FN}$ & 5118 & 9354 & 11101 & 3925 & 8484 & 3783 & 4121 \\
\hline Recall & 0.620 & 0.306 & 0.176 & 0.709 & 0.370 & 0.719 & 0.694 \\
\hline Precision & 0.462 & 0.701 & 0.893 & 0.248 & 0.756 & 0.588 & 0.706 \\
\hline F1 Score & 0.529 & 0.426 & 0.294 & 0.367 & 0.497 & 0.647 & 0.700 \\
\hline Specificity & 0.941 & 0.989 & 0.998 & 0.826 & 0.990 & 0.959 & 0.977 \\
\hline NPV & 0.968 & 0.946 & 0.937 & 0.972 & 0.951 & 0.977 & 0.975 \\
\hline Accuracy & 0.917 & 0.938 & 0.937 & 0.817 & 0.944 & 0.941 & 0.955 \\
\hline Kappa & 0.485 & 0.399 & 0.276 & 0.288 & 0.471 & 0.615 & 0.676 \\
\hline MCC & 0.491 & 0.437 & 0.381 & 0.343 & 0.505 & 0.619 & 0.676 \\
\hline Excerpt 2 & Wendt & Martin & Wamsley & Bodizs & Moelle & Devuyst & DETOKS \\
\hline TP & 9881 & 7954 & 0 & 11045 & 7006 & 9862 & 10307 \\
\hline TN & 320771 & 339807 & 344818 & 268002 & 340517 & 336140 & 339091 \\
\hline FP & 24754 & 5718 & 707 & 77523 & 5008 & 9385 & 6433 \\
\hline $\mathrm{FN}$ & 4594 & 6521 & 14475 & 3430 & 7469 & 4613 & 4168 \\
\hline Recall & 0.683 & 0.549 & 0.000 & 0.763 & 0.484 & 0.681 & 0.71 \\
\hline Precision & 0.285 & 0.582 & 0.000 & 0.125 & 0.583 & 0.512 & 0.62 \\
\hline F1 Score & 0.402 & 0.565 & 0.000 & 0.214 & 0.529 & 0.585 & 0.67 \\
\hline Specificity & 0.928 & 0.983 & 0.998 & 0.776 & 0.986 & 0.973 & 0.98 \\
\hline NPV & 0.986 & 0.981 & 0.960 & 0.987 & 0.979 & 0.986 & 0.99 \\
\hline Accuracy & 0.918 & 0.966 & 0.958 & 0.775 & 0.965 & 0.961 & 0.97 \\
\hline Kарра & 0.366 & 0.548 & -0.004 & 0.156 & 0.511 & 0.565 & 0.65 \\
\hline MCC & 0.407 & 0.548 & -0.009 & 0.246 & 0.514 & 0.571 & 0.65 \\
\hline Excerpt 3 & Wendt & Martin & Wamsley & Bodizs & Moelle & Devuyst & DETOKS \\
\hline TP & 1005 & 680 & 12 & 0.00 & 0 & 1400 & 1707 \\
\hline TN & 86050 & 87234 & 86741 & 0.00 & 87717 & 86882 & 86772 \\
\hline FP & 1667 & 483 & 976 & 0.00 & 0 & 835 & 944 \\
\hline FN & 1278 & 1603 & 2271 & 0.00 & 2283 & 883 & 576 \\
\hline Recall & 0.440 & 0.298 & 0.005 & 0.00 & 0.000 & 0.613 & 0.748 \\
\hline Precision & 0.376 & 0.585 & 0.012 & 0.00 & 0.000 & 0.626 & 0.644 \\
\hline F1 Score & 0.406 & 0.395 & 0.007 & 0.00 & 0.000 & 0.620 & 0.692 \\
\hline Specificity & 0.981 & 0.994 & 0.989 & 0.00 & 1.000 & 0.990 & 0.989 \\
\hline NPV & 0.985 & 0.982 & 0.974 & 0.00 & 0.975 & 0.990 & 0.993 \\
\hline Accuracy & 0.967 & 0.977 & 0.964 & 0.00 & 0.975 & 0.981 & 0.983 \\
\hline Kappa & 0.389 & 0.384 & -0.008 & 0.00 & 0.000 & 0.610 & 0.683 \\
\hline MCC & 0.390 & 0.407 & -0.009 & 0.00 & 0.000 & 0.610 & 0.685 \\
\hline Excerpt 5 & Wendt & Martin & Wamsley & Bodizs & Moelle & Devuyst & DETOKS \\
\hline TP & 10461 & 9672 & 0 & 14779 & 0 & 10332 & 14164 \\
\hline TN & 330466 & 333514 & 337779 & 295704 & 340039 & 335566 & 333646 \\
\hline FP & 9573 & 6525 & 2260 & 44335 & 0 & 4473 & 6392 \\
\hline $\mathrm{FN}$ & 9500 & 10289 & 19961 & 5182 & 19961 & 9629 & 5797 \\
\hline Recall & 0.524 & 0.485 & 0.000 & 0.740 & 0.000 & 0.518 & 0.710 \\
\hline Precision & 0.522 & 0.597 & 0.000 & 0.250 & 0.000 & 0.698 & 0.689 \\
\hline F1 Score & 0.523 & 0.535 & 0.000 & 0.374 & 0.000 & 0.594 & 0.699 \\
\hline Specificity & 0.972 & 0.981 & 0.993 & 0.870 & 1.000 & 0.987 & 0.981 \\
\hline NPV & 0.972 & 0.970 & 0.944 & 0.983 & 0.945 & 0.972 & 0.983 \\
\hline Accuracy & 0.947 & 0.953 & 0.938 & 0.862 & 0.945 & 0.961 & 0.966 \\
\hline Kappa & 0.495 & 0.511 & -0.011 & 0.317 & 0.000 & 0.574 & 0.681 \\
\hline MCC & 0.495 & 0.514 & -0.019 & 0.377 & 0.000 & 0.581 & 0.681 \\
\hline Excerpt 6 & Wendt & Martin & Wamsley & Bodizs & Moelle & Devuyst & DETOKS \\
\hline TP & 12810 & 11724 & 0 & 17988 & 11928 & 13381 & 15435 \\
\hline TN & 327975 & 332715 & 336704 & 288379 & 331078 & 332761 & 332467 \\
\hline FP & 9588 & 4848 & 859 & 49184 & 6485 & 4802 & 5095 \\
\hline $\mathrm{FN}$ & 9627 & 10713 & 22437 & 4449 & 10509 & 9056 & 7002 \\
\hline Recall & 0.571 & 0.523 & 0.000 & 0.802 & 0.532 & 0.596 & 0.688 \\
\hline Precision & 0.572 & 0.707 & 0.000 & 0.268 & 0.648 & 0.736 & 0.752 \\
\hline F1 Score & 0.571 & 0.601 & 0.000 & 0.401 & 0.584 & 0.659 & 0.718 \\
\hline Specificity & 0.972 & 0.986 & 0.997 & 0.854 & 0.981 & 0.986 & 0.985 \\
\hline NPV & 0.971 & 0.969 & 0.938 & 0.985 & 0.969 & 0.974 & 0.979 \\
\hline Accuracy & 0.947 & 0.957 & 0.935 & 0.851 & 0.953 & 0.962 & 0.966 \\
\hline Kappa & 0.543 & 0.579 & -0.005 & 0.340 & 0.559 & 0.639 & 0.701 \\
\hline MCC & 0.543 & 0.586 & -0.013 & 0.407 & 0.562 & 0.643 & 0.701 \\
\hline
\end{tabular}

